\title{
Single-particle transfer and nuclear supersymmetry
}

\author{
J. Barea, ${ }^{1}$ R. Bijker, ${ }^{2}$ A. Frank ${ }^{2,3}$ and G. Loyola ${ }^{2}$ \\ ${ }^{1}$ Departamento de Física Atómica, Molecular y Nuclear, Facultad de Física, Universidad de Sevilla, Apdo. 1065, 41080 Sevilla, Spain \\ ${ }^{2}$ Instituto de Ciencias Nucleares, Universidad Nacional Autónoma de México, Apartado Postal 70-543, 04510 México, D.F., \\ Mexico \\ ${ }^{3}$ Centro de Ciencias Físicas, Universidad Nacional Autónoma de México, Apartado Postal 139-B, 62251 Cuernavaca, Morelos, Mexico
}

(Received 28 May 2001; published 20 November 2001)

\begin{abstract}
Transfer reactions constitute a stringent test for nuclear supersymmetry, a theory that simultaneously describes neighboring nuclei with bosonic and fermionic character. We construct and analytically evaluate onenucleon transfer matrix elements between supersymmetric partners with the U(6/4) case as an example, and stress the need for a careful treatment of bosonic and fermionic operators in the construction of mixed tensor operators.
\end{abstract}

DOI: $10.1103 /$ PhysRevC.64.064313

PACS number(s): 21.10.Jx, 21.60.Ev, 21.60.Fw, 12.60.Jv

Recently the supersymmetric classification of nuclear levels in the Pt and Au isotopes has been reexamined by taking advantage of the significant improvements in experimental capabilities developed in the last decade. High resolution transfer experiments with protons and polarized deuterons have led to strong evidence for the existence of supersymmetry (SUSY) in atomic nuclei. The experiments include high resolution transfer experiments to ${ }^{196} \mathrm{Au}$ at TU/LMU München [1,2], and in-beam $\gamma$ ray and conversion electron spectroscopy following the reactions ${ }^{196} \operatorname{Pt}(d, 2 n)$ and ${ }^{196} \mathrm{Pt}(p, n)$ at the cyclotrons of PSI and Bonn [3]. These studies have achieved an improved classification of states in ${ }^{195} \mathrm{Pt}$ and ${ }^{196} \mathrm{Au}$ that give further support to the original ideas [4-6] and extend and refine previous experimental work $[7-9]$ in this research area.

In the context of the interacting boson model (IBM) [10] and its extensions [11], Iachello and co-workers proposed that Bose-Fermi symmetries $\mathrm{U}(6) \otimes \mathrm{U}(\Omega)$ can be embedded into a graded Lie algebra $u(6 / \Omega)$ in order to unify even-even and even-odd nuclei $[12,13]$ in a supersymmetric framework. The supersymmetric irreducible representation $[\mathcal{N}\}$ encompasses the even-even nucleus with $\mathcal{N}$ bosons and the oddeven nucleus with $\mathcal{N}-1$ bosons and the odd fermion. This idea was later extended by Van Isacker and co-workers to the case where neutron and proton bosons are distinguished [6], predicting in this way a correlation among quartets of nuclei, the even-even partner with $N_{\nu}+N_{\pi}$ bosons, the odd-proton and odd-neutron neighbors with $N_{\nu}+N_{\pi}-1$ bosons, and the odd-odd nucleus with $N_{\nu}+N_{\pi}-2$ bosons.

While this simultaneous description and classification have been shown to be fulfilled to a good approximation for the quartet of nuclei ${ }^{194} \mathrm{Pt},{ }^{195} \mathrm{Au},{ }^{195} \mathrm{Pt}$, and ${ }^{196} \mathrm{Au}$, there are important predictions still not fully verified by experiments. These tests involve the transfer reaction intensities among the supersymmetric partners. In this Brief Report we discuss the example of proton transfer between the SUSY partners ${ }^{194} \mathrm{Pt}$ and ${ }^{195} \mathrm{Au}$ [e.g., through the $(\vec{d}, n)$ stripping reaction] in the $U(6 / 4)$ scheme. We emphasize that special care has to be taken in the theoretical analysis because of phase ambiguities in the $\mathrm{SO}(6)$ classification [14] of boson and fermion tensor operators.

First, we briefly review the $U(6 / 4)$ supersymmetry. For the sake of simplicity, just as in the original papers $[12,13,15]$, we do not distinguish between neutron and proton bosons. The simultaneous description of ${ }^{194} \mathrm{Pt}$ and ${ }^{195} \mathrm{Au}$ nuclei in the SUSY scheme assumes that the odd-proton hole in ${ }^{195} \mathrm{Au}$ can only occupy the $2 d_{3 / 2}$ orbit. The U(6/4) dynamical supersymmetry involves the group chain

$\mid \begin{array}{ccccccc}\mathrm{U}(6 / 4) & \supset & \mathrm{U}^{B}(6) & \otimes & \mathrm{U}^{F}(4) & \supset & \mathrm{SO}^{B}(6) \\ {[\mathcal{M}\}} & & {[N]} & & \left\{1^{M}\right\} & & (\Sigma, 0,0)\end{array}$

The corresponding Hamiltonian, neglecting terms that contribute only to the binding energy, is of the form

$$
H=A C_{2 \mathrm{SO}^{B}(6)}+B C_{2 \operatorname{Spin}(6)}+C C_{2 \operatorname{Spin}(5)}+D C_{2 \operatorname{Spin}(3)},
$$

which leads to an analytic form for the energy as a function of the quantum numbers

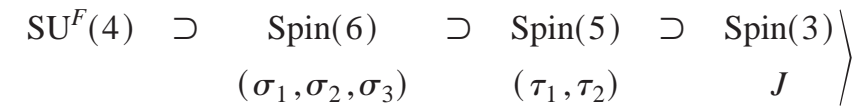

$$
\begin{aligned}
E= & A \Sigma(\Sigma+4)+B\left[\sigma_{1}\left(\sigma_{1}+4\right)+\sigma_{2}\left(\sigma_{2}+2\right)+\sigma_{3}^{2}\right] \\
& +C\left[\tau_{1}\left(\tau_{1}+3\right)+\tau_{2}\left(\tau_{2}+1\right)\right]+D J(J+1) .
\end{aligned}
$$

The coefficients $A, B, C$, and $D$ are determined in a simultaneous fit of the excitation energies of even-even and oddeven nuclei that belong to the same supermultiplet. In addition to energies, in the case of a dynamical supersymmetry closed expressions can be derived for other observables as 
well, such as electromagnetic intensities and single-particle transfer reaction strengths. In the next section we concentrate on the latter, and in particular on the transfer between the even and odd members of the supermultiplet.

The single-particle transfer operator that is commonly used in the interacting boson-fermion model (IBFM) has been derived semimicroscopically in the seniority scheme [16]. Although strictly speaking this derivation is only valid in the vibrational regime, it has been used for deformed nuclei as well. An alternative method is based on symmetry considerations. It consists of expressing the single-particle transfer operator in terms of tensor operators under the subgroups that appear in the group chain of a dynamical (super)symmetry $[15,17]$.

For single-particle transfer between different supermultiplets of $\mathrm{U}(6 / 4)$ characterized by $[\mathcal{N}\}$ and $[\mathcal{N}+1\}$, the transfer operator, to lowest order, is given by $a_{3 / 2, m}^{\dagger}$, which by construction transforms as a $\mathrm{SU}(4)$ tensor operator

$$
P_{\frac{3}{2}, m}^{\dagger}=\alpha a_{\frac{3}{2}, m}^{\dagger}=\alpha T_{\left(\frac{1}{2}, \frac{1}{2}\right), \frac{3}{2}, m}^{\left(\frac{1}{2}, \frac{1}{2}, \frac{1}{2}\right)}
$$

Here the upper indices denote $\left(\sigma_{1}, \sigma_{2}, \sigma_{3}\right)$, which specify the tensorial properties under $\operatorname{Spin}(6)$ which is isomorphic to $\mathrm{SU}(4)$ and $\mathrm{SO}(6)[13,15]$. Similarly, the lower indices represent $\left(\tau_{1}, \tau_{2}\right), J$, and $M$, which define the transformation properties under $\operatorname{Spin}(5)$, Spin(3), and $\operatorname{Spin}(2)$, respectively. For the inverse process we have

$$
\widetilde{P} \frac{3}{2}, m=\alpha \widetilde{a} \frac{3}{2}, m=\alpha T_{\left(\frac{1}{2}, \frac{1}{2}\right), \frac{3}{2}, m}^{\left(\frac{1}{2}, \frac{1}{2},-\frac{1}{2}\right)},
$$

with $\tilde{a}_{j, m}=(-1)^{j-m} a_{j,-m}$.

The most stringent test of supersymmetries, however, is provided by the single-particle transfer between different members of the same supermultiplet. The transfer operator in this case involves the interchange of a boson and a fermion operator,

$$
\begin{aligned}
& P_{\frac{3}{2}, m}^{\dagger}=\sum_{l=0,2} \alpha_{l}\left(b_{l}^{\dagger} \times \widetilde{a}_{3 / 2}\right)_{m}^{(3 / 2)}, \\
& \widetilde{P}_{\frac{3}{2}}, m=\sum_{l=0,2} \alpha_{l}\left(a_{\frac{3}{2}}^{\dagger} \times \widetilde{b}_{l}\right)_{m}^{(3 / 2)},
\end{aligned}
$$

with $\widetilde{b}_{l, m}=(-1)^{l-m} b_{l,-m}$. Here we have introduced the notation $b_{0}^{\dagger}=s^{\dagger}$ and $b_{2}^{\dagger}=d^{\dagger}$. The operators of Eq. (6) are tensor operators under $\operatorname{Spin}(5)$ and $\operatorname{Spin}(3)$, but not under $\operatorname{Spin}(6)$.

Whereas the fermion creation and annihilation operators of Eqs. (4) and (5) transform as tensor operators, the transformation properties of the boson creation and annihilation operators depend on the realization of the so(6) algebra. Group theoretically, the standard realization of the orthogonal subalgebra is given in terms of the antisymmetric generators

$$
\begin{aligned}
& \bar{Q}_{\mu}^{(2)}=-i\left(s^{\dagger} \times \widetilde{d}-d^{\dagger} \times \widetilde{s}\right)_{\mu}^{(2)}, \\
& G_{\mu}^{(\lambda)}=\left(d^{\dagger} \times \widetilde{d}\right)_{\mu}^{(\lambda)} \quad(\lambda=1,3) .
\end{aligned}
$$

The transformation properties of the boson creation and annihilation operators under $\mathrm{SO}(6)$ can be investigated by considering the rotation

$$
\mathcal{R}(\theta)=\exp \left(-i \theta \bar{Q}_{0}^{(2)}\right) .
$$

Since $\mathcal{R}(\theta)$ transforms the creation and annihilation operators in the same way,

$$
\begin{aligned}
& \mathcal{R}(\theta)\left(\begin{array}{c}
s^{\dagger} \\
d_{0}^{\dagger}
\end{array}\right) \mathcal{R}^{-1}(\theta)=\left(\begin{array}{rr}
\cos \theta & \sin \theta \\
-\sin \theta & \cos \theta
\end{array}\right)\left(\begin{array}{l}
s^{\dagger} \\
d_{0}^{\dagger}
\end{array}\right), \\
& \mathcal{R}(\theta)\left(\begin{array}{c}
\tilde{s} \\
\widetilde{d}_{0}
\end{array}\right) \mathcal{R}^{-1}(\theta)=\left(\begin{array}{rr}
\cos \theta & \sin \theta \\
-\sin \theta & \cos \theta
\end{array}\right)\left(\begin{array}{c}
\tilde{s} \\
\widetilde{d}_{0}
\end{array}\right),
\end{aligned}
$$

the annihilation operators have the same tensorial character as the creation operators,

$$
b_{2 \tau, m}^{\dagger}, \widetilde{b}_{2 \tau, m}: T_{(\tau, 0), 2 \tau, m}^{(1,0,0)} . \quad(\tau=0,1) .
$$

The $\mathrm{SO}(6)$ isoscalar factors that are relevant for the $\operatorname{Spin}(6)$ limit can be derived in the standard way by evaluating the matrix elements of the boson-fermion quadrupolequadrupole interaction [15], provided that one uses the antisymmetric quadrupole operator of Eq. (7). The matrix elements of the latter were derived in Ref. [14]. It is important to note that the $\mathrm{SO}(6)$ isoscalar factors are related, but not equivalent to the expansion coefficients $\xi$ of Refs. $[15,18]$ :

$$
\begin{aligned}
& \left\langle\begin{array}{cc|c}
(N, 0,0) & \left(\frac{1}{2}, \frac{1}{2}, \frac{1}{2}\right) & \left(N \pm \frac{1}{2}, \frac{1}{2}, \mp \frac{1}{2}\right) \\
(\tau, 0), L & \left(\frac{1}{2}, \frac{1}{2}\right), \frac{3}{2} & \left(\tau+\frac{1}{2}, \frac{1}{2}\right), J
\end{array}\right) \\
& =-i \xi_{N \pm \frac{1}{2}, \tau+\frac{1}{2}, J}^{N, \tau, L} \\
& \left\langle\begin{array}{cc|c}
(N, 0,0) & \left(\frac{1}{2}, \frac{1}{2}, \frac{1}{2}\right) & \left(N \pm \frac{1}{2}, \frac{1}{2}, \mp \frac{1}{2}\right) \\
(\tau, 0), L & \left(\frac{1}{2}, \frac{1}{2}\right), \frac{3}{2} & \left(\tau-\frac{1}{2}, \frac{1}{2}\right), J
\end{array}\right\rangle \\
& =\xi_{N \pm \frac{1}{2}, \tau-\frac{1}{2}, J}^{N, \tau, L}
\end{aligned}
$$

With these isoscalar factors we can construct the singleparticle transfer operators that connect different members of the same supermultiplet according to their tensorial character. We find

$$
\begin{aligned}
& T_{\left(\frac{1}{2}, \frac{1}{2}\right), \frac{3}{2}, m}^{\left(\sigma_{1}, \sigma_{2}, \sigma_{3}\right)} \\
& \quad \sum_{\tau=0,1}\left\langle\begin{array}{cc|c}
(1,0,0) & \left(\frac{1}{2}, \frac{1}{2}, \frac{1}{2}\right) & \left(\sigma_{1}, \sigma_{2}, \sigma_{3}\right) \\
(\tau, 0), 2 \tau & \left(\frac{1}{2}, \frac{1}{2}\right), \frac{3}{2} & \left(\frac{1}{2}, \frac{1}{2}\right), \frac{3}{2}
\end{array}\right\rangle \\
& \quad \times\left(\widetilde{b}_{2 \tau} \times a_{3 / 2}^{\dagger}\right)_{m}^{(3 / 2)} .
\end{aligned}
$$


For the two allowed representations $\left(\sigma_{1}, \sigma_{2}, \sigma_{3}\right)$ of $\operatorname{Spin}(6)$ this gives

$$
\begin{aligned}
& T_{\left(\frac{1}{2}, \frac{1}{2}\right), \frac{3}{2}, m}^{\left(\frac{1}{2}, \frac{1}{2},-\frac{1}{2}\right)}=-i \sqrt{\frac{1}{6}}\left(\tilde{s} \times a_{\frac{3}{2}}^{\dagger}\right)_{m}^{\left(\frac{3}{2}\right)}+\sqrt{\frac{5}{6}}\left(\tilde{d} \times a_{\frac{3}{2}}^{\dagger}\right)_{m}^{\left(\frac{3}{2}\right)}, \\
& T_{\left(\frac{1}{2}, \frac{1}{2}\right), \frac{3}{2}, m}^{\left(\frac{3}{2}, \frac{1}{2}, \frac{1}{2}\right)}=i \sqrt{\frac{5}{6}}\left(\tilde{s} \times a_{\frac{3}{2}}^{\dagger}\right)_{m}^{\left(\frac{3}{2}\right)}+\sqrt{\frac{1}{6}}\left(\tilde{d} \times a_{\frac{3}{2}}^{\dagger}\right)_{m}^{\left(\frac{3}{2}\right)} .
\end{aligned}
$$

However, the realization commonly used in the IBM is in terms of the symmetric quadrupole operator

$$
\begin{aligned}
& Q_{\mu}^{(2)}=\left(s^{\dagger} \times \widetilde{d}+d^{\dagger} \times \widetilde{s}\right)_{\mu}^{(2)}, \\
& G_{\mu}^{(\lambda)}=\left(d^{\dagger} \times \widetilde{d}\right)_{\mu}^{(\lambda)} \quad(\lambda=1,3) .
\end{aligned}
$$

Both realizations, Eqs. (7) and (14), lead to identical energy spectra, but the corresponding wave functions differ in relative phases. The transformation properties of the boson creation and annihilation operators under this realization of $\mathrm{SO}(6)$ can be investigated by considering the rotation

$$
\mathcal{R}(\theta)=\exp \left(-i \theta Q_{0}^{(2)}\right) .
$$

In this case, we find the transformation properties

$$
\begin{gathered}
\mathcal{R}(\theta)\left(\begin{array}{c}
i s^{\dagger} \\
d_{0}^{\dagger}
\end{array}\right) \mathcal{R}^{-1}(\theta)=\left(\begin{array}{rr}
\cos \theta & \sin \theta \\
-\sin \theta & \cos \theta
\end{array}\right)\left(\begin{array}{c}
i s^{\dagger} \\
d_{0}^{\dagger}
\end{array}\right), \\
\mathcal{R}(\theta)\left(\begin{array}{c}
-i \tilde{s} \\
\widetilde{d}_{0}
\end{array}\right) \mathcal{R}^{-1}(\theta)=\left(\begin{array}{rr}
\cos \theta & \sin \theta \\
-\sin \theta & \cos \theta
\end{array}\right)\left(\begin{array}{c}
-i \tilde{s} \\
\widetilde{d}_{0}
\end{array}\right) .
\end{gathered}
$$

The transformation properties of Eq. (16) can thus be expressed as the $\mathrm{SO}(6)$ rotations of Eq. (9) by carrying out the canonical transformation

$$
\begin{gathered}
\tilde{s} \rightarrow i \tilde{s}, \\
s^{\dagger} \rightarrow-i s^{\dagger}, \\
\widetilde{d}_{m} \rightarrow \widetilde{d}_{m}, \\
d_{m}^{\dagger} \rightarrow d_{m}^{\dagger} .
\end{gathered}
$$

This has important consequences for the transfer operators. The tensor operators for single-particle transfer for the realization of Spin(6) with the symmetric boson quadrupole operator and the antisymmetric fermion quadrupole operator [15] can now be obtained by applying the inverse of the canonical transformation of Eq. (17) to the tensor operators of Eq. (13). Accordingly we find

$$
\begin{aligned}
& T_{\left(\frac{1}{2}, \frac{1}{2}\right), \frac{3}{2}, m}^{\left(\frac{1}{2}, \frac{1}{2},-\frac{1}{2}\right)}=-\sqrt{\frac{1}{6}}\left(\tilde{s} \times a_{\frac{3}{2}}^{\dagger}\right)_{m}^{\left(\frac{3}{2}\right)}+\sqrt{\frac{5}{6}}\left(\tilde{d} \times a_{\frac{3}{2}}^{\dagger}\right)_{m}^{\left(\frac{3}{2}\right)}, \\
& T_{\left(\frac{1}{2}, \frac{1}{2}\right), \frac{3}{2}, m}^{\left(\frac{3}{2}, \frac{1}{2}, \frac{1}{2}\right)}=\sqrt{\frac{5}{6}}\left(\tilde{s} \times a_{\frac{3}{2}}^{\dagger}\right)_{m}^{\left(\frac{3}{2}\right)}+\sqrt{\frac{1}{6}}\left(\tilde{d} \times a_{\frac{3}{2}}^{\dagger}\right)_{m}^{\left(\frac{3}{2}\right)},
\end{aligned}
$$

and for the inverse process

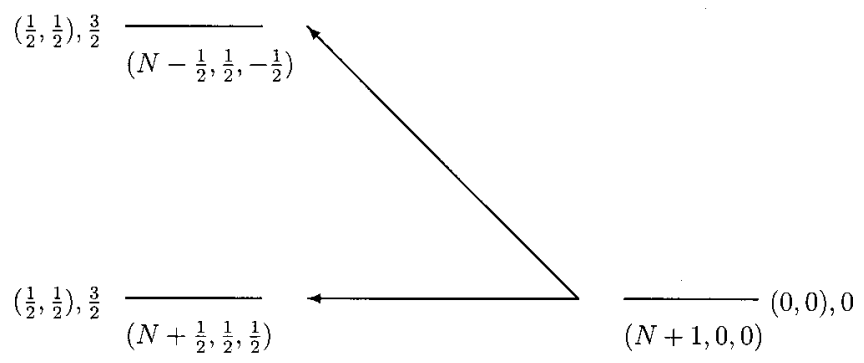

FIG. 1. Allowed single-particle transfer reactions for transitions from even-even to odd-even nuclei in $\mathrm{U}(6 / 4)$.

$$
\begin{aligned}
& T_{\left(\frac{1}{2}, \frac{1}{2}\right), \frac{3}{2}, m}^{\left(\frac{1}{2}, \frac{1}{2}, \frac{1}{2}\right)}=-\sqrt{\frac{1}{6}}\left(s^{\dagger} \times \tilde{a}_{3 / 2}\right)_{m}^{\left(\frac{3}{2}\right)}+\sqrt{\frac{5}{6}}\left(d^{\dagger} \times \tilde{a}_{3 / 2}\right)_{m}^{\left(\frac{3}{2}\right)}, \\
& T_{\left(\frac{1}{2}, \frac{1}{2}\right), \frac{3}{2}, m}^{\left(\frac{3}{2}, \frac{1}{2},-\frac{1}{2}\right)}=\sqrt{\frac{5}{6}}\left(s^{\dagger} \times \tilde{a}_{3 / 2}\right)_{m}^{\left(\frac{3}{2}\right)}+\sqrt{\frac{1}{6}}\left(d^{\dagger} \times \tilde{a}_{3 / 2}\right)_{m}^{\left(\frac{3}{2}\right)} .
\end{aligned}
$$

We note, as is well known, that the annihilation operator $d_{m}$ by itself is not a spherical tensor operator. This led to the introduction of the "tilde" operator $\widetilde{d}_{m}=(-1)^{m} d_{-m}$, which does have the same tensor properties as the creation operator $d_{m}^{\dagger}$. In the present case, we have shown that with the usual realization of the so(6) algebra used in the IBM, the boson creation and annihilation operators by themselves do not form a $\mathrm{SO}(6)$ tensor, but rather

$$
\left(\begin{array}{c}
i s^{\dagger} \\
d_{m}^{\dagger}
\end{array}\right),\left(\begin{array}{c}
-i \tilde{s} \\
\widetilde{d}_{m}
\end{array}\right):\left(\begin{array}{c}
T_{(0,0), 0,0}^{(1,0,0)} \\
T_{(1,0), 2, m}^{(1,0,0)}
\end{array}\right) .
$$

The use of tensor operators to describe single-particle transfer reactions in the supersymmetry scheme has the advantage of giving rise to selection rules and closed expressions for the spectroscopic factors. Figure 1 shows the allowed transitions for the transfer operators of Eq. (18) that describe the single-particle transfer from the ground state $|(N+1,0,0),(0,0), 0\rangle$ of the even-even nucleus to the oddeven nucleus belonging to the same supermultiplet $[N+1\}$. Both operators have the same transformation character under $\operatorname{Spin}(5)$ and $\operatorname{Spin}(3)$, and therefore can only excite states with $\left(\tau_{1}, \tau_{2}\right)=\left(\frac{1}{2}, \frac{1}{2}\right)$ and $J=\frac{3}{2}$. The operators differ in their Spin(6) selection rules. Whereas the first operator can only excite the ground state band of the odd-even nucleus with $\left(\sigma_{1}, \sigma_{2}, \sigma_{3}\right)=\left(N+\frac{1}{2}, \frac{1}{2}, \frac{1}{2}\right)$, the second one allows a transition to an excited band as well $\left(N \pm \frac{1}{2}, \frac{1}{2}, \pm \frac{1}{2}\right)$.

Figure 2 shows the allowed transitions for the inverse process, i.e., the single-particle transfer from the ground state $\left|\left(N+\frac{1}{2}, \frac{1}{2}, \frac{1}{2}\right),\left(\frac{1}{2}, \frac{1}{2}\right), \frac{3}{2}\right\rangle$ of the odd-even nucleus to the eveneven nucleus by the transfer operators of Eq. (19). Both operators satisfy the same selection rules under $\operatorname{Spin}(5)$ and $\operatorname{Spin}(3)$, which permit the excitation of states with $\left(\tau_{1}, \tau_{2}\right)$, $J=(0,0), 0$ or $(1,0), 2$. According to the $\operatorname{Spin}(6)$ selection rules, the first operator can only excite the ground state band of the even-even nucleus with $\left(\sigma_{1}, \sigma_{2}, \sigma_{3}\right)=(N+1,0,0)$, whereas the second one allows a transition to an excited band as well $(N \pm 1,0,0)$. 


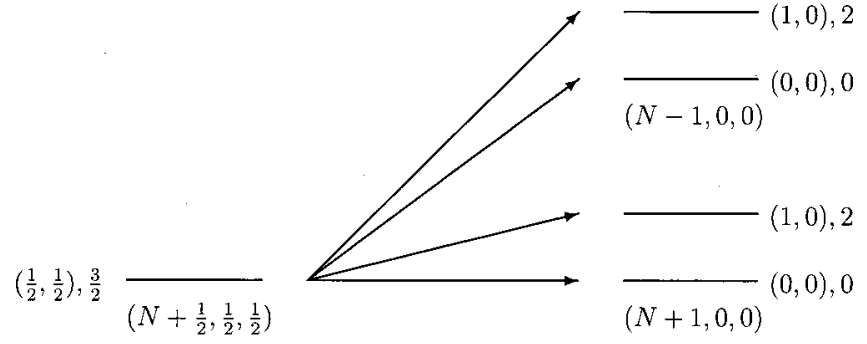

FIG. 2. Allowed single-particle transfer reactions for transitions from odd-even to even-even nuclei in $\mathrm{U}(6 / 4)$.

In Table I, we present the corresponding matrix elements of the transfer operators of Eqs. (18) and (19). An analysis of spectroscopic factors for proton pickup and stripping reactions between the pair of nuclei ${ }^{192} \mathrm{Os}$ and ${ }^{193}$ Ir shows that the operators $T^{\left(\frac{1}{2}, \frac{1}{2}, \mp \frac{1}{2}\right)}$ of Eqs. (18) and (19) decribe the main features of the data [15]. For the SUSY partners ${ }^{194} \mathrm{Pt}$ and ${ }^{195} \mathrm{Au}$, there is to the best of our knowledge only data available for the proton stripping reactions ${ }^{194} \mathrm{Pt}(\alpha, t){ }^{195} \mathrm{Au}$ and ${ }^{194} \mathrm{Pt}\left({ }^{3} \mathrm{He}, d\right){ }^{195} \mathrm{Au}$ [19]. The $J=3 / 2$ ground state of ${ }^{195} \mathrm{Au}$ is excited strongly with $C^{2} S=0.175$, whereas the first excited $J=3 / 2$ state is excited weakly with $C^{2} S=0.019$. In the SUSY scheme, the latter state is assigned as a member of the ground state band with $\left(\tau_{1}, \tau_{2}\right)=(5 / 2,1 / 2)$. Therefore the one-proton transfer to this state is forbidden by the $\operatorname{Spin}(5)$ selection rule of the tensor operators of Eq. (18). The relatively small strength of excited $J=3 / 2$ states suggests that, also in this case, the operator $T^{\left(\frac{1}{2}, \frac{1}{2},-\frac{1}{2}\right)}$ of Eqs. (18) can be used to describe the data.

Finally, we note the relevance of tensor operators in the study of supersymmetric multiphonon structures [20], as well as in the construction of supersymmetric "ladder" operators that convert the eigenstates of an even-even nucleus into the eigenstates of the odd-even nucleus [21].

The recent measurements of the spectroscopic properties of the odd-odd nucleus ${ }^{196} \mathrm{Au}$ have rekindled the interest in nuclear supersymmetry. The available data on the spectroscopy of the quartet of nuclei ${ }^{194} \mathrm{Pt},{ }^{195} \mathrm{Au},{ }^{195} \mathrm{Pt}$, and ${ }^{196} \mathrm{Au}$ can, to a good approximation, be described in terms of the $\mathrm{U}(6 / 4) \otimes U(6 / 12)$ supersymmetry. However, there is still another important set of experiments which can further test the predictions of the supersymmetry scheme. These involve single-particle transfer reactions between nuclei belonging to the same supermultiplet, in particular between the even-odd and odd-odd members of the supersymmetric quartet. Theoretically, these transfers are described by the supersymmetric generators that change a boson into a fermion, or vice versa.

We investigated in detail the example of proton transfer between the SUSY partners ${ }^{194} \mathrm{Pt}$ and ${ }^{195} \mathrm{Au}$ in $\mathrm{U}(6 / 4)$, and

TABLE I. Matrix elements of one-proton transfer operators in the Spin(6) limit for transitions (a) from even-even to odd-even and (b) from odd-even to even-even. (a) The ground state of the even-even nucleus has $|i\rangle=|[N+1\},[N+1],\{0\},(N+1,0,0),(0,0), 0\rangle$, and (b) the ground state of the odd-even nucleus $|i\rangle$ $=\left|[N+1\},[N],\{1\},\left(N+\frac{1}{2}, \frac{1}{2}, \frac{1}{2}\right),\left(\frac{1}{2}, \frac{1}{2}\right), \frac{3}{2}\right\rangle$. For the final states we only show the labels $\langle f|$ $=\left\langle\left(\sigma_{1}, \sigma_{2}, \sigma_{3}\right),\left(\tau_{1}, \tau_{2}\right), J\right|$.

(a) $\langle f| \quad\left\langle f|| T_{\left(\frac{1}{2}, \frac{1}{2}\right), \frac{3}{2}}^{\left(\frac{1}{2}, \frac{1}{2},-\frac{1}{2}\right)} \| i\right\rangle \quad\left\langle f|| T_{\left(\frac{1}{2}, \frac{1}{2}\right), \frac{3}{2}}^{\left(\frac{3}{2}, \frac{1}{2}\right)} \| i\right\rangle$

\begin{tabular}{ccc}
$\left\langle\left(N+\frac{1}{2}, \frac{1}{2}, \frac{1}{2}\right),\left(\frac{1}{2}, \frac{1}{2}\right), \frac{3}{2}\right|$ & $-\sqrt{\frac{2(N+1)}{3}}$ & $-\frac{N+5}{N+2} \sqrt{\frac{8(N+1)}{15}}$ \\
$\left\langle\left(N-\frac{1}{2}, \frac{1}{2},-\frac{1}{2}\right),\left(\frac{1}{2}, \frac{1}{2}\right), \frac{3}{2}\right|$ & 0 & $\frac{\sqrt{6 N(N+1)(N+4) / 5}}{N+2}$ \\
\hline
\end{tabular}

(b) $\langle f|$

$\left\langle f\left\|T_{\left(\frac{1}{2}, \frac{1}{2}\right), \frac{3}{2}}^{\left(\frac{1}{2}, \frac{1}{2}, \frac{1}{2}\right)}\right\| i\right\rangle$

$\left\langle f\left\|T_{\left(\frac{1}{2}, \frac{1}{2}\right), \frac{3}{2}}^{\left(\frac{3}{2}, \frac{1}{2},-\frac{1}{2}\right)}\right\| i\right\rangle$

$\begin{array}{lcc}\langle(N+1,0,0),(0,0), 0| & -\sqrt{\frac{2(N+1)}{3}} & -\frac{N+5}{N+2} \sqrt{\frac{8(N+1)}{15}} \\ \langle(N+1,0,0),(1,0), 2| & \sqrt{\frac{2(N+5)}{3}} & \frac{N-1}{N+2} \sqrt{\frac{8(N+5)}{15}} \\ \langle(N-1,0,0),(0,0), 0| & 0 & -\frac{1}{N+2} \sqrt{\frac{6 N(N+3)(N+4)}{5(N+1)}} \\ \langle(N-1,0,0),(1,0), 2| & 0 & \frac{1}{N+2} \sqrt{\frac{6(N-1) N(N+4)}{5(N+1)}}\end{array}$


pointed out that special care should be taken in the theoretical analysis because of phase ambiguities in the so(6) algebra. The latter arise because in the usual realization of the Spin(6) algebra the symmetric boson quadrupole operator is combined with the antisymmetric fermion quadrupole operator. We have shown explicitly how in this case the transfer operators can be classified according to their tensorial character under $\operatorname{Spin}(6) \supset \operatorname{Spin}(5) \supset \operatorname{Spin}(3)$. This makes it possible to study the corresponding selection rules and derive their matrix elements.

The problem of the phase ambiguities arises especially for coupled systems, such as the neutron-proton IBM, the IBFM, the neutron-proton IBFM, and especially the extension to odd-odd nuclei. As an example, the odd-odd nucleus ${ }^{196} \mathrm{Au}$ is described as a system with four neutron bosons, one proton boson, one neutron and one proton. Moreover, the phase ambiguities not only occur in the $\mathrm{SO}(6)$ limit of the IBM, but also in the SU(3) limit [22]. For the deriva- tion of selection rules and the evaluation of spectroscopic factors and transfer intensities, it is crucial to use consistent conventions in the construction of the appropiate tensor operators for electromagnetic transitions and one-particle transfer reactions. Further work in this direction is in progress [23]. To conclude, we emphasize the need for new experiments taking advantage of the new experimental capabilities [1-3] and suggest that particular attention should be paid to one-nucleon transfer reactions between the SUSY partners ${ }^{194} \mathrm{Pt},{ }^{195} \mathrm{Au},{ }^{195} \mathrm{Pt}$, and ${ }^{196} \mathrm{Au}$, since such experiments provide the most accurate tests of nuclear supersymmetry.

This work was supported in part by CONACyT under Project Nos. 32416-E and 32397-E, by DPAGA-UNAM under Project No. IN106400, and by DGICYT under Project No. PB98-1111. One of us (J.B.) is very grateful to his hosts at the ICN-UNAM.
[1] A. Metz, J. Jolie, G. Graw, R. Hertenberger, J. Gröger, C. Günther, N. Warr, and Y. Eisermann, Phys. Rev. Lett. 83, 1542 (1999).

[2] A. Metz, Y. Eisermann, A. Gollwitzer, R. Hertenberger, B.D. Valnion, G. Graw and J. Jolie, Phys. Rev. C 61, 064313 (2000).

[3] J. Gröger, J. Jolie, R. Krücken, C.W. Beausang, M. Caprio, R.F. Casten, J. Cederkall, J.R. Cooper, F. Corminboeuf, L. Genilloud, G. Graw, C. Günther, M. de Huu, A.I. Levon, A. Metz, J.R. Novak, N. Warr, and T. Wendel, Phys. Rev. C 62, 064304 (2000).

[4] A.B. Balantekin, I. Bars, R. Bijker, and F. Iachello, Phys. Rev. C 27, 1761 (1983).

[5] H.Z. Sun, A. Frank, and P. van Isacker, Phys. Rev. C 27, 2430 (1983).

[6] P. Van Isacker, J. Jolie, K. Heyde, and A. Frank, Phys. Rev. Lett. 54, 653 (1985).

[7] A. Mauthofer, K. Stelzer, J. Gerl, Th.W. Elze, Th. Happ, G. Eckert, T. Faestermann, A. Frank, and P. van Isacker, Phys. Rev. C 34, 1958 (1986).

[8] J. Jolie, U. Mayerhofer, T. von Egidy, H. Hiller, J. Klora, H. Lindner, and H. Trieb, Phys. Rev. C 43, R16 (1991).

[9] G. Rotbard, G. Berrier, M. Vergnes, S. Fortier, J. Kalifa, J.M.
Maison, L. Rosier, J. Vernotte, P. van Isacker, and J. Jolie, Phys. Rev. C 47, 1921 (1993).

[10] F. Iachello and A. Arima, The Interacting Boson Model (Cambridge University Press, Cambridge, 1987).

[11] F. Iachello and P. van Isacker, The Interacting Boson-Fermion Model (Cambridge University Press, Cambridge, 1991).

[12] F. Iachello, Phys. Rev. Lett. 44, 772 (1980).

[13] A.B. Balantekin, I. Bars, and F. Iachello, Phys. Rev. Lett. 47, 19 (1981); Nucl. Phys. A370, 284 (1981).

[14] P. Van Isacker, A. Frank, and J. Dukelsky, Phys. Rev. C 31, 671 (1985).

[15] F. Iachello and S. Kuyucak, Ann. Phys. (N.Y.) 136, 19 (1981).

[16] O. Scholten, Prog. Part. Nucl. Phys. 14, 189 (1985).

[17] R. Bijker and F. Iachello, Ann. Phys. (N.Y.) 161, 360 (1985).

[18] S. Kuyucak, Ph.D. thesis, Yale University, 1982.

[19] M.L. Munger and R.J. Peterson, Nucl. Phys. A303, 199 (1978).

[20] K.-H. Kim, T. Otsuka, A. Gelberg, P. von Brentano, and P. van Isacker, Phys. Rev. Lett. 76, 3514 (1996).

[21] R.V. Jolos, P. von Brentano, A. Gelberg, K.-H. Kim, and T. Otsuka, Phys. Lett. B 430, 1 (1998).

[22] A.M. Shirokov, N.A. Smirnova, Yu.F. Smirnov, O. Castaños, and A. Frank, Phys. At. Nucl. 63, 760 (2000).

[23] J. Barea, G. Loyola, R. Bijker, and A. Frank (unpublished). 